\title{
Study on a Conceptual Mobile Memory of Interface Designation
}

\author{
Yanxin Xie, Yanpeng Li, Bo Song, and Chunyu Jiang \\ Jilin Agricultural Science and Technology University, Jilin, China, 132101
}

Keywords: personalized design; storage device; transplantation

\begin{abstract}
In the age of data explosion, people have higher requirements for large data storage design, with sufficient storage capacity, high speed, and portability, also a new design for the personalized design of storage devices. Memory is widely used and the market demand is large. Therefore, the development of memory technology is facing new opportunities for diversification. "Faster computing speed and more storage space" is the direction of research and development by scientists around the world in the field of computer storage, and the industry competition for mobile storage devices has been accelerated. Therefore, this design proposes a concept-based mobile memory interface design, the peripheral circuit is simple and the performance ratio is high, which can be ported to a single-chip microcomputer system or an embedded system.
\end{abstract}

\section{Introduction}

In April 2018, Wei Zhang and Peng Zhou, a professor at the School of Microelectronics at Fudan University, realized a subversive two-dimensional semiconductor quasi-non-volatile storage prototype device, created a third type of storage technology, and Fudan University initiated research and development of a third type of storage technology. Can reduce energy consumption to achieve data validity period clipping. This new feature can first greatly reduce storage power consumption in high-speed memory, especially for some large computers, and the energy saving will be considerable.

Recently, at the "China Communications Integrated Circuit Technology and Application Symposium and the 2nd JJN International Integrated Circuit Industry Development Summit Forum", Academician Ming Liu, researcher, doctoral tutor of the Institute of Microelectronics of the Chinese Academy of Sciences, and director of the Key Laboratory of Microelectronics Devices and Integration Technology of the Chinese Academy of Sciences, Delivered a keynote speech entitled "Memory technology development situation and opportunities". The speech talked about 8 kinds of memory (5 kinds of traditional +3 kinds of new models), introduced the development of traditional memory technology and packaging technology, the principle and advantages and disadvantages of new sensors, and the memory field and research and industry status quo that China has vigorously developed [1]-[2].

\section{Current Status of Memory Development}

In 1967, Fairchild invented the first 64-bit read-only memory. In the same year, the Bell Laboratory was born Flash. In 1970 Intel launched the first SRAM and the first EEPROM. However, ordinary users did not become aware of the importance of memory until the large-scale application of flash memory in the 1990s. Memory has now prevailed for more than 50 years. Today, in 1970 Intel launched SRAM and DRAM. In 1984, Masuoka and others first proposed flash memory. In 1988, Intel and Toshiba introduced NOR and NAND flash respectively. Sumi launched FeRAM in 1994. After 1995, Motoraola and other companies introduced MRAM and PRAM. According to the characteristics of memory storage information, it is divided into two categories: volatile and non-volatile. The volatile memory is SRAM and DRAM; There are many types of non-volatile memory, which are divided into two categories. The first category is traditional: Flash memory(FLASH), SONOS, ferroelectric memory(FRAM), phase transition memory(OUM), magnetic memory(MRAM) and resistance memory(RRAM) and so on. The second type is the new 
memory PRAM, MRAM and RRAM. as shown in figure 1, In the future, the development of storage devices will pay more attention to the two major development directions of process and peripheral packaging. Such as with the rapid development of semiconductor technology, from 8F2(feature size) to 4F2. All mainstream DRAM production is set below 20nm. In addition to the continuous reduction of the process, packaging technology also provides another way for the development of memory, and China's vigorously developed memory DRAM and NAND Flash [3]-[7].

In addition to the shrinking process, packaging technology also provides another way for the development of DRAM, such as HBM (high bandwidth memory) and HMC (mixed storage cube). Fig. 1 is a diagram of memory classification.

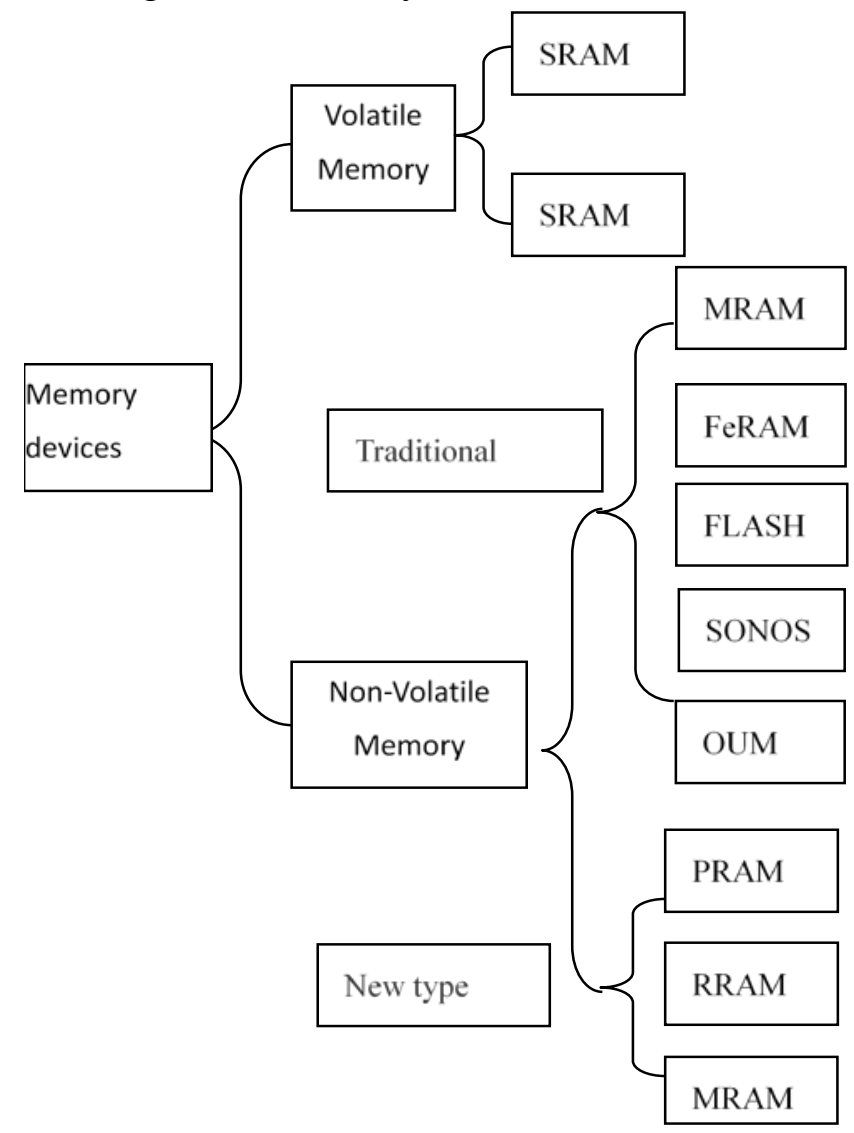

Fig.1 The diagram of Memory classification.

Today on a chip (SOC) storage system, there are a number of problems: the first of which is that it consumed most of the power consumption of the system, and the second is the system program takes microcontroller system storage space is bigger, cause a large load of equipment; Thirdly, the sealing difference causes the internal structure material to be oxidized, thus affecting the reliability of the connection. The fourth is that some interfaces, when connected with the computer, appear to be loose, which also affects the reliability of the connection. Therefore, a new conceptual load small mobile storage and mobile memory architecture is required.

\section{The Working Principle of Memory}

Because of the emergence of semiconductor technology, storage technology has been continuously upgraded, and this magnetic storage technology is the most common in all kinds of mobile storage technology, which has been continued until now. Removable magnetic storage devices work by actuators, which are also mechanical devices connected to computers. Reading and writing information can be achieved through the drive. 


\section{The Construction Principle of Memory}

Removable storage device external structure: the last layer has an iron oxide coating. This oxide is ferromagnetic material, and if it is exposed to a magnetic field, it will be permanently magnetized. This medium is commonly referred to as a disk or disk. The drive uses a high speed rotation to store information through the medium on its magnetic head. Every head has a package of iron core coil tiny electromagnet it will exert a magnetic flux to the oxide coating on medium, oxide can permanently "remember" it felt flux effect. When reading and writing, the data signal is propagated along the circle, and then a magnetic field is generated in the iron core. Interference fringes are formed in the space between the head and the medium under the effect of the flux effect. This interference effect creates a bridge between the gaps, and then the oxide on the magnetized medium. When the driver reads the data, the read head will apply a magnetic field that can pass through the gap, and a changing magnetic field will be generated in the iron core, and an electrical signal will be generated on the coil. The signal is then transmitted to the computer in binary form [8]-[10].

\section{Unique Appearance Design}

This design is mainly to make up for the shortage, putting forward a conceptual memory architecture, as shown in figure 2, two sealing parts are added at the peripheral interface. During the work, two sealing parts are connected with a locking part, and two locking parts are provided with a small clamping plate at the transmission connecting part. The clamping plate is provided with several many angles. The pin plate is provided at the port part of the sealing plate, and the sealing plate is disposed at.

The outer opening of the device through the pin shaft. The pin shaft is designed with a gear on the outside, and the gear is connected with the torsion spring at the outer opening; in order to prevent looseness when being used, Therefore, a rack is provided, which is engaged with the gear on the side, which is convenient for reading data. A right-angled trapezoid is arranged at the end of the rack, a roller is arranged under the driving block, and a transmission lever is provided on the roller interface piece. The lower side of the transmission rod is fixedly connected with the clamp plate, and a spring is arranged between the plate and the inner wall of the external frame at the same time.

Fig.2 is a diagram of mobile storage device structure.

Among them: 1 -the sealing part, 2 -rack, 3 - the guide block, 4 - drive blocks, 5 - splint, 6 - the scroll wheel, 7 - the transmission rod, 8 - the spring, 9 - frame ,10 - magnet.

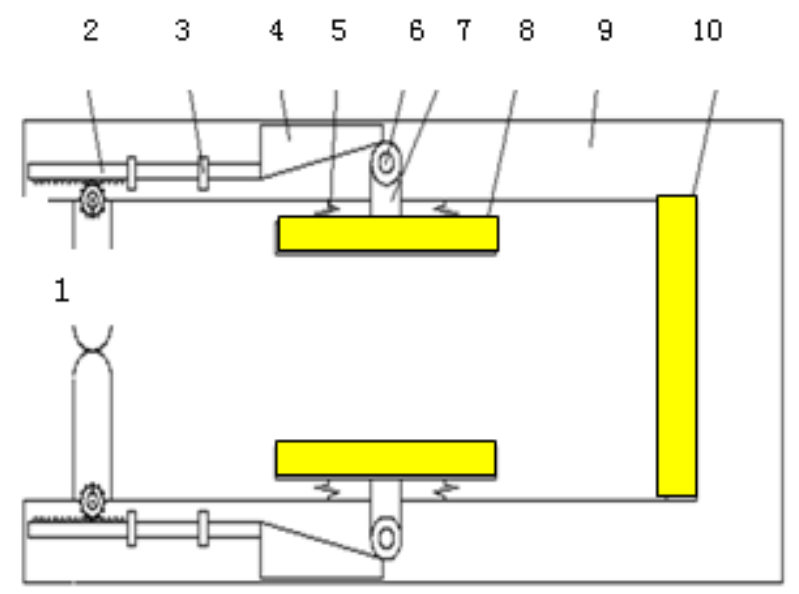

Fig.2 The diagram of mobile storage device structure.

\section{When the mobile storage is inserted}

When the mobile storage is inserted into the device (as shown in figure 3), it will push the two 
sealing plates to rotate. The sealing plate will control the pin rotation and the pin axis will also control the gear rotation. Then the movement of the control side of the rack, gear and rack control trapezoidal move, because the right Angle trapezoid drive block, will control wheel rotating at the same time, makes the transmission rod move down, which can control two plywood for mobile storage device clamp; When the mobile storage device inserted in place after two splint clamping the mobile storage equipment at the same time, the magnet will absorb of mobile: storage devices, to realize the reliable fixed mobile storage equipment.Fig.2 is a Working principle diagram of mobile storage device.

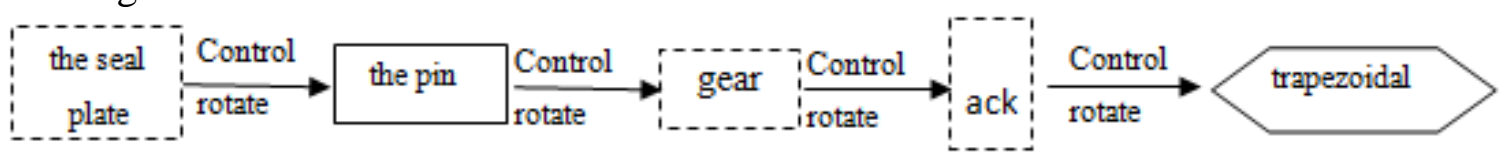

Fig.3 The Working principle diagram of mobile storage device

\section{When the Mobile Storage is Pulled out}

When removing the mobile storage device, the drive block, the sealing plate and the splint will be reset due to the action of spring and torsion spring, and the sealing inside the outer frame can be realized to prevent internal oxidation.

\section{Layers of Safety Braking}

There are two guide blocks on the rack, and the rack passes through two guide blocks to realize the reliable horizontal movement of the rack.

The outer layer has a layer of shielding.

The outer frame adopts the encapsulation design method, the sum of the length of two sealing plates is equal to the aperture of the outer frame, to prevent the oxidation of several multi-angle in the interior.

\section{Conclusion}

Due to the problem of materials and artificial design reasons, the product is relatively thick, so the successor will continuously improve the appearance and volume of the product, so as to improve the product.

\section{References}

[1] Wei Zhan, Peng Zhou A semi-floating gate memory based on van der Waals heterostructures for quasi-nonvolatile applications[J]. Nature Nanotechnology 2017

[2] http://www.ednchina.com/news/article/20170727memory

[3] Beijing Zhongyuan Zhisheng Market Research co., LTD. Research Report on the Development Trend and Competitive Strategy of China Mobile U Disk Industry in 2016-2021 [R]. Beijing,2016.6.

[4] Shenzhen Zhongqi Zhiye Investment Consulting co., LTD.2016-2021 Mobile Storage Equipment Market Prospect Forecast and Investment Planning Analysis Report [R]. Shenzhen,2016.10.

[5] http://www.eepw.com.cn/article/262534.htm,

[6] http://www.21ic.com/app/mcu/201208/140905.htm, 2012-08-31

[7] Chen Dan, Yang Yongchun, Li Enlong, and Shi Kunbo. The Use of Mobile Smart Devices for Beijing Residents' Multi-task Shopping Behavior and Commercial Micro-location [J]. Chinese Science: Earth Science,2018(03):353-365. 
[8] Anmer, L., \& Nashchold, F. (2000). Attaining the international development targets: will growth be enough? DFID working paper. DFID, London Hazell and Haddad, 2001.

[9] Yang Jifan, Huang Rongquan. Mobile News Generation and Development in the Era of Mobile Media [J/OL]. New Media Research,2018(04)

[10] Feng Xu, Hua Qing. Design and Implementation of a User Interface Description Language for Mobile Devices [J/OL]. Computer Engineering: [2018 (03) 1-7.

[11] Blundell, R., Bond, S., 1998. Initial conditions and moment restrictions in dynamic panel data models. Journal of Econometrics 87 (1),115-143. 